Supporting Information of

\title{
Preserving Symmetry and Degeneracy in the Localized Orbital Scaling Correction Approach
}

\author{
Neil Qiang $\mathrm{Su}^{1}$, Aaron Mahler ${ }^{2}$ and Weitao Yang ${ }^{1,3}$ \\ ${ }^{1}$ Department of Chemistry, Duke University, Durham, North Carolina 27708, USA ${ }^{2}$ Department \\ of Physics, Duke University, Durham, North Carolina 27708, USA ${ }^{3}$ Key Laboratory of Theoretical \\ Chemistry of Environment, School of Chemistry and Environment, South China Normal \\ University, Guangzhou 510006, China
}

(Dated: February 1, 2020) 


\section{Relation between symmetry and degeneracy}

All the symmetry operations associated with the geometry of a particular system, have the property of permuting atoms in identical environment in the system and form a symmetry group (point group or space group). In commonly used density functional approximations (DFAs), such as local density approximation (LDA), generalized gradient approximation (GGA), and the Hartree-Fock approximation, the one-electron Hamiltonian, $\hat{h}$, which depends on the density and the geometry (due to nuclear-attraction operator), commutes with symmetry operators $\left\{\hat{R}_{k}\right\}$, which usually forms a subgroup of molecular symmetry group; namely

$$
\hat{R}_{k} \hat{h}=\hat{h} \hat{R}_{k}
$$

Let $\left\{\varepsilon_{p}\right\}$ and $\left\{\varphi_{p}(\mathbf{r})\right\}$ be the eigenvalues and eigenstates of $\hat{h}$ respectively. Now applying a symmetry operator, $\hat{R}_{k}$, on both side of the eigenequation,

$$
\hat{h} \varphi_{p}(\mathbf{r})=\varepsilon_{p} \varphi_{p}(\mathbf{r})
$$

we obtain

$$
\hat{R}_{k} \hat{h} \varphi_{p}(\mathbf{r})=\hat{h} \hat{R}_{k} \varphi_{p}(\mathbf{r})=\varepsilon_{p} \hat{R}_{k} \varphi_{p}(\mathbf{r})
$$

Thus, $\hat{R}_{k} \varphi_{p}(\mathbf{r})$ is also an eigenstate of $\hat{h}$ associated with eigenvalue $\varepsilon_{p}$. If $\varphi_{p}(\mathbf{r})$ is not a degenerate state, then it can differ from $\hat{R}_{k} \varphi_{p}(\mathbf{r})$ only by a constant factor, i.e. $\hat{R}_{k} \varphi_{p}(\mathbf{r})=$ $C \varphi_{p}(\mathbf{r})$. If $\varphi_{p}(\mathbf{r})$ and $\varphi_{q}(\mathbf{r})$ form a pair of doubly degenerate states, then $\hat{R}_{k} \varphi_{p}(\mathbf{r})$ can be obtained by linear combination of $\varphi_{p}(\mathbf{r})$ and $\varphi_{q}(\mathbf{r})$, i.e.

$$
\hat{R}_{k} \varphi_{p}(\mathbf{r})=\varphi_{p}(\mathbf{r}) U_{p p}^{k}+\varphi_{q}(\mathbf{r}) U_{q p}^{k} . \text { or. } \hat{R}_{k}\left[\varphi_{p}(\mathbf{r}), \varphi_{q}(\mathbf{r})\right]=\left[\varphi_{p}(\mathbf{r}), \varphi_{q}(\mathbf{r})\right] \mathrm{U}^{\mathrm{k}}
$$

Thus, $\varphi_{p}(\mathbf{r})$ and $\varphi_{q}(\mathbf{r})$ carry a two-dimensional irreducible representation of $\left\{\hat{R}_{k}\right\}$, and $\mathrm{U}^{\mathrm{k}}$ is the matrix representation of the symmetry operator $\hat{R}_{k}$ within the subspace of $\varphi_{p}(\mathbf{r})$ and 
$\varphi_{q}(\mathbf{r})$. Similarly, if $\varphi_{p}(\mathbf{r}), \varphi_{q}(\mathbf{r}), \ldots$ are $n$-fold degenerate states (excluding accidental degeneracy), then they carry a $n$-dimensional irreducible representation of $\left\{\hat{R}_{k}\right\}$. Therefore, the symmetry of the system decides the degeneracy of the one-electron orbitals once the relation of Eq. (S1) holds. Each set of degenerate states corresponds to an irreducible representation of the symmetry subgroup to which the system belongs, with the degeneracy equal to the dimension of the representation.

Table S1: Character table for $D_{6 h}$ point group

\begin{tabular}{c|cccccccccccc}
\hline$D_{6 h}$ & $E$ & $2 C_{6}$ & $2 C_{3}$ & $C_{2}$ & $3 C_{2}^{\prime}$ & $3 C_{2}^{\prime \prime}$ & $I$ & $2 S_{3}$ & $2 S_{6}$ & $\sigma_{h}$ & $3 \sigma_{d}$ & $3 \sigma_{v}$ \\
\hline $\mathrm{A}_{1 \mathrm{~g}}$ & 1 & 1 & 1 & 1 & 1 & 1 & 1 & 1 & 1 & 1 & 1 & 1 \\
$\mathrm{~B}_{1 \mathrm{u}}$ & 1 & -1 & 1 & -1 & 1 & -1 & -1 & 1 & -1 & 1 & -1 & 1 \\
$\mathrm{~A}_{2 \mathrm{~g}}$ & 1 & 1 & 1 & 1 & -1 & -1 & 1 & 1 & 1 & 1 & -1 & -1 \\
$\mathrm{~B}_{2 \mathrm{u}}$ & 1 & -1 & 1 & -1 & -1 & 1 & -1 & 1 & -1 & 1 & 1 & -1 \\
$\mathrm{~B}_{1 \mathrm{~g}}$ & 1 & -1 & 1 & -1 & 1 & -1 & 1 & -1 & 1 & -1 & 1 & -1 \\
$\mathrm{~A}_{1 \mathrm{u}}$ & 1 & 1 & 1 & 1 & 1 & 1 & -1 & -1 & -1 & -1 & -1 & -1 \\
$\mathrm{~A}_{2 \mathrm{u}}$ & 1 & 1 & 1 & 1 & -1 & -1 & -1 & -1 & -1 & -1 & 1 & 1 \\
$\mathrm{~B}_{2 \mathrm{~g}}$ & 1 & -1 & 1 & -1 & -1 & 1 & 1 & -1 & 1 & -1 & -1 & 1 \\
$\mathrm{E}_{1 \mathrm{u}}$ & 2 & 1 & -1 & -2 & 0 & 0 & -2 & -1 & 1 & 2 & 0 & 0 \\
$\mathrm{E}_{2 \mathrm{~g}}$ & 2 & -1 & -1 & 2 & 0 & 0 & 2 & -1 & -1 & 2 & 0 & 0 \\
$\mathrm{E}_{1 \mathrm{~g}}$ & 2 & 1 & -1 & -2 & 0 & 0 & 2 & 1 & -1 & -2 & 0 & 0 \\
$\mathrm{E}_{2 \mathrm{u}}$ & 2 & -1 & -1 & 2 & 0 & 0 & -2 & 1 & 1 & -2 & 0 & 0 \\
\hline
\end{tabular}

Table S2: Character table for $D_{3 h}$ point group

\begin{tabular}{c|cccccc}
\hline$D_{3 h}$ & $E$ & $2 C_{3}$ & $3 C_{2}^{\prime}$ & $2 S_{3}$ & $\sigma_{h}$ & $3 \sigma_{v}$ \\
\hline $\mathrm{A}_{1}^{\prime}$ & 1 & 1 & 1 & 1 & 1 & 1 \\
$\mathrm{~A}_{1}^{\prime \prime}$ & 1 & 1 & 1 & -1 & -1 & -1 \\
$\mathrm{~A}_{2}^{\prime}$ & 1 & 1 & -1 & 1 & 1 & -1 \\
$\mathrm{~A}_{2}^{\prime \prime}$ & 1 & 1 & -1 & -1 & -1 & 1 \\
$\mathrm{E}^{\prime}$ & 2 & -1 & 0 & -1 & 2 & 0 \\
$\mathrm{E}^{\prime \prime}$ & 2 & -1 & 0 & 1 & -2 & 0 \\
\hline
\end{tabular}

Table S3: Character table for $D_{3}$ point group

\begin{tabular}{c|ccc}
\hline$D_{3}$ & $E$ & $2 C_{3}$ & $3 C_{2}^{\prime}$ \\
\hline $\mathrm{A}_{1}$ & 1 & 1 & 1 \\
$\mathrm{~A}_{2}$ & 1 & 1 & -1 \\
$\mathrm{E}$ & 2 & -1 & 0 \\
\hline
\end{tabular}


Thus, in order to derive the orbital degeneracy of the system under study, usually it is not necessary to use the full symmetry group, its subgroup that satisfies Eq. S1 is sufficient. Taking benzene $\left(\mathrm{C}_{6} \mathrm{H}_{6}\right)$ as example, the full symmetry group is the point group $D_{6 h}$, see Tab. S1 for the character table. It includes 8 one-dimensional representations and 4 two-dimensional representations, where labels $\mathrm{A}$ and $\mathrm{B}$ are for one-dimensional irreducible representations while E for two-dimensional representations. Regarding the symmetry operators in the tables, $E$ is identity operation, $C_{n}$ is rotation by $2 \pi / n$ angle, $I$ is inversion operation, $S_{n}$ is improper rotation (rotation by $2 \pi / n$ angle and reflection in the plane perpendicular to the axis), $\sigma_{h}$ is horizontal reflection plane (perpendicular to the principal axis), $\sigma_{v}$ is vertical reflection plane (contains the principal axis), and $\sigma_{d}$ is diagonal reflection plane (contains the principal axis and bisects the angle between two $C_{2}$ axes perpendicular to the principal axis). $D_{3 h}$ is a subgroup of $D_{6 h}$, see Tab. S2 for the character table. By removing those symmetry operators that are not included in $D_{3 h}$ from Tab. S1, the rest of the table shows that the 12 irreducible representations reduce to the 6 irreducible representations of $D_{3 h}$. The correspondence of the irreducible representations between $D_{6 h}$ and $D_{3 h}$ are shown in Tab. S4. Similarly, the 6 irreducible representations of $D_{3 h}$ can further reduce to the 3 irreducible representations of $D_{3}$. Tab. S4 shows that all the four two-dimensional representations of $D_{6 h}$ reduce to the two-dimensional representations of $D_{3 h}$ and $D_{3}$, which means that the degeneracy is not destroyed. The key symmetry element that guarantees the degeneracy of the states in $\mathrm{C}_{6} \mathrm{H}_{6}$ is $C_{3}$, which is possessed by all the three point groups mentioned here.

Table S4: Correspondence of the irreducible representations among $D_{6 h}, D_{3 h}$ and $D_{3}$

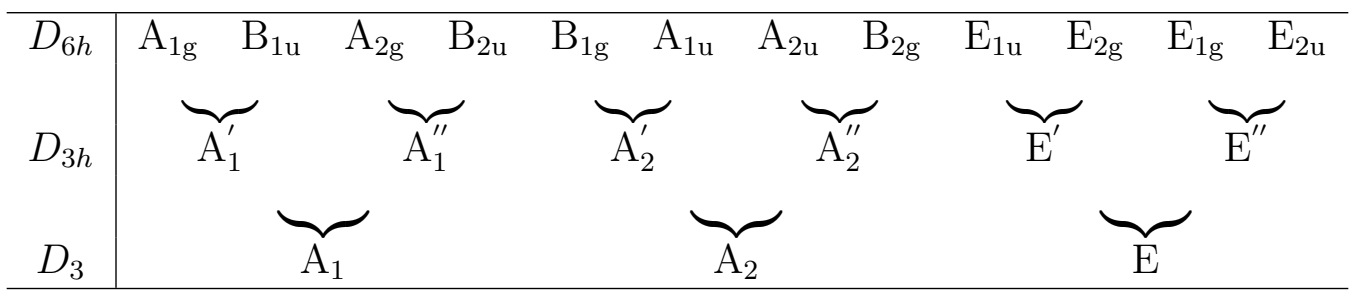




\section{Algorithm for orbital localization}

The objective function for orbital localization in this work consists of a physical space part and an energy space part, which takes the form of

$$
F=(1-\lambda) \sum_{p} R_{p p p p}+\lambda C \sum_{p} H_{p p p p}
$$

where

$$
R_{p q r s}=\left\langle\phi_{p}(1) \phi_{r}(2)\left|\left(\mathbf{r}_{1}-\mathbf{r}_{2}\right)^{2}\right| \phi_{q}(1) \phi_{s}(2)\right\rangle
$$

and

$$
H_{p q r s}=\left\langle\phi_{p}(1) \phi_{r}(2)\left|[h(1)-h(2)]^{2}\right| \phi_{q}(1) \phi_{s}(2)\right\rangle .
$$

$h$ is the one-electron Hamiltonian of the parent DFA. The localized orbitals (LOs) are obtained via

$$
\phi_{p}(\mathbf{r})=\sum_{q} U_{p q} \varphi_{q}(\mathbf{r})
$$

and the U matrix can be obtained by minimizing Eq. (S5).

Here shows the algorithm of minimizing the objective function of Eq. (S5), or other objective functions based on $R_{\text {pppp }}$ and $H_{\text {pppp }}\left(F=(1-\lambda) \sum_{p} f_{r}\left(R_{\text {pppp }}\right)+\lambda C \sum_{p} f_{h}\left(H_{\text {pppp }}\right)\right.$ ), through a series of $2 \times 2$ rotations, which can be efficiently computed through an easily coded parallel implementation.

1. Initialization:

(a) Initialize LOs:

$$
\phi_{p}(\mathbf{r})=\varphi_{p}(\mathbf{r})
$$


(b) Construct some matrices:

$$
\begin{aligned}
\overline{\mathbf{r}}_{p q} & =\left\langle\phi_{p}|\mathbf{r}| \phi_{q}\right\rangle, \\
{\overline{r^{2}}}_{p q} & =\left\langle\phi_{p}\left|r^{2}\right| \phi_{q}\right\rangle, \\
\bar{h}_{p q} & =\left\langle\phi_{p}|h| \phi_{q}\right\rangle=\varepsilon_{p} \delta_{p q}, \\
{\overline{h^{2}}}_{p q} & =\left\langle\phi_{p}\left|h^{2}\right| \phi_{q}\right\rangle=\varepsilon_{p}^{2} \delta_{p q} .
\end{aligned}
$$

As these matrices are symmetric, so only the elements of the lower triangular matrices are needed to be computed.

(c) Compute the objective function, where $R_{p q r s}$ of Eq. (S6) and $H_{p q r s}$ of Eq. (S7) can be obtained via

$$
\begin{aligned}
& R_{p q r s}=\bar{r}_{p q}^{2} \delta_{r s}+\bar{r}_{r s}^{2} \delta_{p q}-2 \overline{\mathbf{r}}_{p q} \cdot \overline{\mathbf{r}}_{r s}, \\
& H_{p q r s}=\bar{h}_{p q}^{2} \delta_{r s}+\bar{h}_{r s}^{2} \delta_{p q}-2 \bar{h}_{p q} \bar{h}_{r s} .
\end{aligned}
$$

2. $2 \times 2$ rotations for the $i$-th iteration:

(a) Rotate a pair of LOs, $\phi_{p}$ and $\phi_{q}$ with $p>q$, via

$$
\begin{gathered}
\phi_{p}^{\prime}=\phi_{p} \cos (\theta)+\phi_{q} \sin (\theta), \\
\phi_{q}^{\prime}=-\phi_{p} \sin (\theta)+\phi_{q} \cos (\theta),
\end{gathered}
$$

where, $\theta$ decides the rotation matrix

$$
U^{p q}=\left(\begin{array}{cc}
\cos (\theta) & \sin (\theta) \\
-\sin (\theta) & \cos (\theta)
\end{array}\right)
$$


(b) Minimize the objective function. The change of the objective function caused by two-orbital rotation is

$$
\begin{aligned}
\Delta F_{p q}(\theta) & =(1-\lambda)\left[f_{r}\left(R_{\text {pppp }}(\theta)\right)+f_{r}\left(R_{q q q q}(\theta)\right)\right]+\lambda C\left[f_{h}\left(H_{p p p p}(\theta)\right)+f_{h}\left(H_{q q q q}(\theta)\right)\right] \\
& -(1-\lambda)\left[f_{r}\left(R_{p p p p}\right)+f_{r}\left(R_{q q q q}\right)\right]-\lambda C\left[f_{h}\left(H_{p p p p}\right)+f_{h}\left(H_{q q q q}\right)\right] .
\end{aligned}
$$

Here

$$
\begin{aligned}
R_{\text {pppp }}(\theta) & =\left\langle\phi_{p}^{\prime}(1) \phi_{p}^{\prime}(2)\left|\left(\mathbf{r}_{1}-\mathbf{r}_{2}\right)^{2}\right| \phi_{p}^{\prime}(1) \phi_{p}^{\prime}(2)\right\rangle \\
& =x^{0}+x^{c 2} \cos (2 \theta)+x^{c 4} \cos (4 \theta)+x^{s 2} \sin (2 \theta)+x^{s 4} \sin (4 \theta) \\
R_{q q q q}(\theta) & =\left\langle\phi_{q}^{\prime}(1) \phi_{q}^{\prime}(2)\left|\left(\mathbf{r}_{1}-\mathbf{r}_{2}\right)^{2}\right| \phi_{q}^{\prime}(1) \phi_{q}^{\prime}(2)\right\rangle \\
& =x^{0}-x^{c 2} \cos (2 \theta)+x^{c 4} \cos (4 \theta)-x^{s 2} \sin (2 \theta)+x^{s 4} \sin (4 \theta)
\end{aligned}
$$

with

$$
\begin{aligned}
x^{0} & =\frac{1}{8}\left(3 R_{p p p p}+2 R_{p p q q}+4 R_{p q p q}+3 R_{q q q q}\right), \\
x^{c 2} & =\frac{1}{2}\left(R_{p p p p}-R_{q q q q}\right), \\
x^{c 4} & =\frac{1}{8}\left(R_{p p p p}-2 R_{p p q q}-4 R_{p q p q}+R_{q q q q}\right), \\
x^{s 2} & =R_{p p p q}+R_{p q q q}, \\
x^{s 4} & =\frac{1}{2}\left(R_{p p p q}-R_{p q q q}\right) .
\end{aligned}
$$


And

$$
\begin{aligned}
H_{p p p p}(\theta) & =\left\langle\phi_{p}^{\prime}(1) \phi_{p}^{\prime}(2)\left|[h(1)-h(2)]^{2}\right| \phi_{p}^{\prime}(1) \phi_{p}^{\prime}(2)\right\rangle \\
& =y^{0}+y^{c 2} \cos (2 \theta)+y^{c 4} \cos (4 \theta)+y^{s 2} \sin (2 \theta)+y^{s 4} \sin (4 \theta), \\
H_{q q q q}(\theta) & =\left\langle\phi_{q}^{\prime}(1) \phi_{q}^{\prime}(2)\left|[h(1)-h(2)]^{2}\right| \phi_{q}^{\prime}(1) \phi_{q}^{\prime}(2)\right\rangle \\
& =y^{0}-y^{c 2} \cos (2 \theta)+y^{c 4} \cos (4 \theta)-y^{s 2} \sin (2 \theta)+y^{s 4} \sin (4 \theta),
\end{aligned}
$$

with

$$
\begin{aligned}
y^{0} & =\frac{1}{8}\left(3 H_{p p p p}+2 H_{p p q q}+4 H_{p q p q}+3 H_{q q q q}\right), \\
y^{c 2} & =\frac{1}{2}\left(H_{p p p p}-H_{q q q q}\right), \\
y^{c 4} & =\frac{1}{8}\left(H_{p p p p}-2 H_{p p q q}-4 H_{p q p q}+H_{q q q q}\right), \\
y^{s 2} & =H_{p p p q}+H_{p q q q}, \\
y^{s 4} & =\frac{1}{2}\left(H_{p p p q}-H_{p q q q}\right) .
\end{aligned}
$$

The 4-index matrices $R_{p q r s}$ and $H_{p q r s}$ can be easily obtained via Eq. (S14) and Eq. (S15), so they donot need to be stored.

As $\Delta F_{p q}(\theta)$ is a simple trigonometric function of $\theta$, it is easy to apply the NewtonRaphson method to find the minimum. We need also the first and second derivatives, i.e.

$$
\begin{aligned}
\frac{d \Delta F_{p q}(d \theta)}{d \theta} & =\frac{\partial \Delta F_{p q}(\theta)}{\partial R_{p p p p}(\theta)} \frac{d R_{p p p p}(\theta)}{d \theta}+\frac{\partial \Delta F_{p q}(\theta)}{\partial R_{q q q q}(\theta)} \frac{d R_{q q q q}(\theta)}{d \theta} \\
& +\frac{\partial \Delta F_{p q}(\theta)}{\partial E_{p p p p}(\theta)} \frac{d E_{p p p p}(\theta)}{d \theta}+\frac{\partial \Delta F_{p q}(\theta)}{\partial E_{q q q q}(\theta)} \frac{d E_{q q q q}(\theta)}{d \theta}
\end{aligned}
$$


and

$$
\begin{aligned}
\frac{d^{2} \Delta F_{p q}(\theta)}{(d \theta)^{2}}= & \frac{\partial \Delta F_{p q}(\theta)}{\partial R_{p p p p}(\theta)} \frac{d^{2} R_{p p p p}(\theta)}{(d \theta)^{2}}+\frac{\partial^{2} \Delta F_{p q}(\theta)}{\left[\partial R_{p p p p}(\theta)\right]^{2}}\left[\frac{d R_{p p p p}(\theta)}{d \theta}\right]^{2} \\
+ & \frac{\partial \Delta F_{p q}(\theta)}{\partial R_{q q q q}(\theta)} \frac{d^{2} R_{q q q q}(\theta)}{(d \theta)^{2}}+\frac{\partial^{2} \Delta F_{p q}(\theta)}{\left[\partial R_{q q q q}(\theta)\right]^{2}}\left[\frac{d R_{q q q q}(\theta)}{d \theta}\right]^{2} \\
& +\frac{\partial \Delta F_{p q}(\theta)}{\partial E_{p p p p}(\theta)} \frac{d^{2} E_{p p p p}(\theta)}{(d \theta)^{2}}+\frac{\partial^{2} \Delta F_{p q}(\theta)}{\left[\partial E_{p p p p}(\theta)\right]^{2}}\left[\frac{d E_{p p p p}(\theta)}{d \theta}\right]^{2} \\
+ & \frac{\partial \Delta F_{p q}(\theta)}{\partial E_{q q q q}(\theta)} \frac{d^{2} E_{q q q q}(\theta)}{(d \theta)^{2}}+\frac{\partial^{2} \Delta F_{p q}(\theta)}{\left[\partial E_{q q q q}(\theta)\right]^{2}}\left[\frac{d E_{q q q q}(\theta)}{d \theta}\right]^{2},
\end{aligned}
$$

with

$$
\begin{aligned}
& \frac{d R_{p p p p}(\theta)}{d \theta}=-2 x^{c 2} \sin (2 \theta)-4 x^{c 4} \sin (4 \theta)+2 x^{s 2} \cos (2 \theta)+4 x^{s 4} \cos (4 \theta) \\
& \frac{d R_{q q q q}(\theta)}{d \theta}=2 x^{c 2} \sin (2 \theta)-4 x^{c 4} \sin (4 \theta)-2 x^{s 2} \cos (2 \theta)+4 x^{s 4} \cos (4 \theta) \\
& \frac{d H_{p p p p}(\theta)}{d \theta}=-2 y^{c 2} \sin (2 \theta)-4 y^{c 4} \sin (4 \theta)+2 y^{s 2} \cos (2 \theta)+4 y^{s 4} \cos (4 \theta) \\
& \frac{d H_{q q q q}(\theta)}{d \theta}=2 y^{c 2} \sin (2 \theta)-4 y^{c 4} \sin (4 \theta)-2 y^{s 2} \cos (2 \theta)+4 y^{s 4} \cos (4 \theta) \\
& \frac{d^{2} R_{p p p p}(\theta)}{(d \theta)^{2}}=-4 x^{c 2} \cos (2 \theta)-16 x^{c 4} \cos (4 \theta)-4 x^{s 2} \sin (2 \theta)-16 x^{s 4} \sin (4 \theta), \\
& \frac{d^{2} R_{q q q q}(\theta)}{(d \theta)^{2}}=4 x^{c 2} \cos (2 \theta)-16 x^{c 4} \cos (4 \theta)+4 x^{s 2} \sin (2 \theta)-16 x^{s 4} \sin (4 \theta) \\
& \frac{d^{2} H_{p p p p}(\theta)}{(d \theta)^{2}}=-4 y^{c 2} \cos (2 \theta)-16 y^{c 4} \cos (4 \theta)-4 y^{s 2} \sin (2 \theta)-16 y^{s 4} \sin (4 \theta), \\
& \frac{d^{2} H_{q q q q}(\theta)}{(d \theta)^{2}}=4 y^{c 2} \cos (2 \theta)-16 y^{c 4} \cos (4 \theta)+4 y^{s 2} \sin (2 \theta)-16 y^{s 4} \sin (4 \theta)
\end{aligned}
$$

As all the derivatives above are easy to calculate, so the minimization can be carried out very fast. Besides, to avoid being trapped in a local minimum, several initial guesses are generated from equidistant points in the interval $[0, \pi]$.

(c) Update the pair of LOs, $\phi_{p}$ and $\phi_{q}$, and some related matrices. The pair of LOs can be updated by

$$
\phi_{p} \leftarrow \phi_{p}^{\prime}, \phi_{q} \leftarrow \phi_{q}^{\prime}
$$


where the rotation matrix $U^{p q}$ of Eq. (S18) can be obtained by inserting the optimized $\theta$. Then, the matrices $\overline{\mathbf{r}}, \overline{r^{2}}, \bar{h}$, and $\overline{h^{2}}$ are updated by applying the rotation $U^{p q}$ to rows and columns $(p, q)$ of all the four matrices.

(d) Repeat (a)-(c) for each pair of LOs, and sum over the changes of the objective function from the rotatin of each pair of LOs,

$$
\Delta F=\sum_{q>p} \Delta F_{p q} .
$$

3. Stop if $\Delta F$ is smaller than a chosen tolerance $\delta$, otherwise go bach to Step 2 with $i=i+1$.

\section{Proof of symmetric $\mathrm{H}_{2}^{+}$orbitalets in LOSC2}

Different from $\mathrm{H}_{2}^{+}$orbitalets of LOSC1 changing from COs at equilibrium bond length to two symmetric LOs at dissociation limit through a series of unsymmetric LOs in middle range, in LOSC2, orbitalets from Eq. (7) go directly from COs to symmetric LOs at some stretched bond length (with the length decided by the parameter $\gamma$ in Eq. (7)). The proof is as below.

For alpha spin, other COs except LUMO are much higher than HOMO in energics, thus only LUMO $\left(\varphi_{2}\right)$ will mix with HOMO $\left(\varphi_{1}\right)$ during the bond dissociation, i.e.

$$
\begin{array}{r}
\phi_{1}=\varphi_{1} \cos (\theta)+\varphi_{2} \sin (\theta), \\
\phi_{2}=-\varphi_{1} \sin (\theta)+\varphi_{2} \cos (\theta) .
\end{array}
$$


The change of the objective function Eq. (7) for the mix of HOMO and LUMO is

$$
\begin{aligned}
\Delta F_{12}(\theta)= & (1-\lambda)\left[R_{1111}(\theta)+R_{2222}(\theta)\right]+\lambda C\left[H_{1111}(\theta)+H_{2222}(\theta)\right] \\
& -(1-\lambda)\left[R_{1111}+R_{2222}\right]-\lambda C\left[H_{1111}+H_{2222}\right] \\
= & 2(1-\lambda)\left[x^{0}+x^{c 4} \cos (4 \theta)+x^{s 4} \sin (4 \theta)\right]+2 \lambda C\left[y^{0}+y^{c 4} \cos (4 \theta)+y^{s 4} \sin (4 \theta)\right] \\
& -(1-\lambda)\left[R_{1111}+R_{2222}\right]-\lambda C\left[H_{1111}+H_{2222}\right] \\
= & z^{0}+z^{c 4} \cos (4 \theta)+z^{s 4} \sin (4 \theta),
\end{aligned}
$$

where

$$
\begin{aligned}
z^{0} & =\frac{1}{4}(1-\lambda)\left(-R_{1111}+2 R_{1122}+4 R_{1212}-R_{2222}\right)+\frac{1}{4} \lambda C\left(-H_{1111}+2 H_{1122}+4 H_{1212}-H_{2222}\right) \\
z^{c 4} & =\frac{1}{4}(1-\lambda)\left(R_{1111}-2 R_{1122}-4 R_{1212}+R_{2222}\right)+\frac{1}{4} \lambda C\left(H_{1111}-2 H_{1122}-4 H_{1212}+H_{2222}\right)
\end{aligned}
$$

$z^{s 4}=(1-\lambda)\left(R_{1112}-R_{1222}\right)+\lambda C\left(H_{1112}-H_{1222}\right)$.

As HOMO and LUMO of alpha spin are symmetric and antisymmetric respectively, by inserting Eq. (S14) and Eq. (S15), it is easy to determine from symmetry that $z^{s 4}$ equates zero along the $\mathrm{H}-\mathrm{H}$ bond dissociation. With this, the change of the objective function with $\theta$ comes only from $z^{c 4} \cos (4 \theta)$. To reach the minimization, $\theta$ should be either 0 for $z^{c 4}<0$, or $\pi / 4$ for $z^{c 4}>0$. Fig. S1 plots $z^{c 4}$ as a function of $\mathrm{H}-\mathrm{H}$ bond length, it shows that the value of $z^{c 4}$ changes from negative to positive at around 2.1, thus orbitalets remain $\operatorname{COs}(\theta=0)$ until the stretched bond length reaches 2.1, after this point, two LOs of 0.5 occupation are obtained from the complete mix of HOMO and LUMO $(\theta=\pi / 4)$. Therefore, $\mathrm{H}_{2}^{+}$orbitalets of LOSC2 change sharply from COs to LOs during $\mathrm{H}-\mathrm{H}$ bond dissociation, which preserves the symmetry of LOs for any bond length. However, based on this set of symmetric orbitalets, it poses a great challenge to construct a proper correction to the total energy so as to obtain 
a dissociation energy curve with continuous derivative.

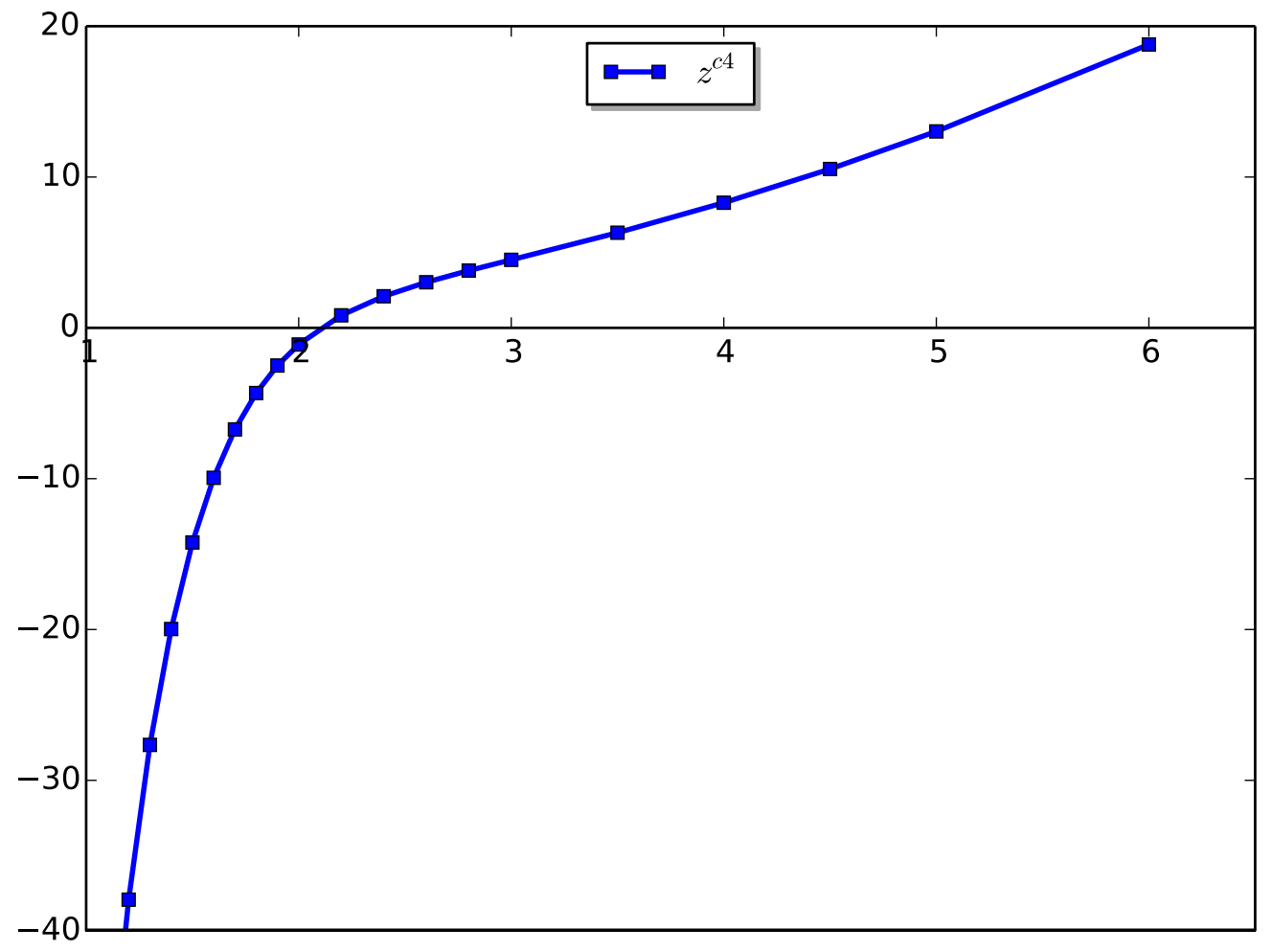

Figure S1: $z^{c 4}$ plotted along the $\mathrm{H}-\mathrm{H}$ bond dissociation for $\mathrm{H}_{2}^{+}$. It changes from negative to positive around 2.1 the units are a.u. and for $\mathrm{y}$ and $\mathrm{x}$ axes respectively.

\section{Relation between symmetry and Bloch theorem}

The Bloch theorem ${ }^{1}$ is very important in the calculation of periodic systems. It states that the wave function of each energy eigenstate in a perfectly periodic potential can be written in the form

$$
\varphi_{n \mathbf{k}}(\mathbf{r})=e^{\mathrm{i} \mathbf{k} . \mathbf{r}} u_{n \mathbf{k}}(\mathbf{r})
$$

where $u_{n \mathbf{k}}(\mathbf{r})$ has the periodicity of the lattice, i.e. $u_{n \mathbf{k}}\left(\mathbf{r}+n_{1} \mathbf{a}_{1}+n_{2} \mathbf{a}_{2}+n_{3} \mathbf{a}_{3}\right)=u_{n \mathbf{k}}(\mathbf{r})$, with the three lattice vectors $\mathbf{a}_{1}, \mathbf{a}_{2}$, and $\mathbf{a}_{3}$, and $n_{1-3}$. In principle, an infinite number of calculations with different wavevectors, k, confined to the first Brillouin zone are required. 
However, since the electronic wave functions will be almost identical for k-points that are sufficiently close, the wave functions over a region of reciprocal space can be well represented by the wave function at a single $\mathbf{k}$-point. Therefore, the Bloch theorem makes the problem of calculating an infinite number of electronic wave functions viable by calculating a finite number of electronic wave functions over an finite number of $\mathbf{k}$-points.

Let $\hat{T}_{\mathbf{n}}$ denote a translation operator that shifts each point in space by the amount of $n_{1} \mathbf{a}_{1}+n_{2} \mathbf{a}_{2}+n_{3} \mathbf{a}_{3}$. As all such translation operators commute with each other then they have common eigenstates, which are Bloch functions with the form of Eq. (S52). For a normal DFA, since the Hamiltonian, $\hat{h}$, is invariant to any of the translations of $n_{1} \mathbf{a}_{1}+n_{2} \mathbf{a}_{2}+n_{3} \mathbf{a}_{3}$, it follows that $\hat{h}$ commutes with each of the translation operator $\hat{T}_{\mathbf{n}}$,

$$
\hat{h} \hat{T}_{\mathbf{n}}=\hat{T}_{\mathbf{n}} \hat{h} .
$$

Therefore, the eigenstates of $\hat{h}$ can be chosen to be eigenstates of all $\hat{T}_{\mathbf{n}}$ simultaneously, which thus makes the Bloch theorem applicable.

As LOs from LOSC1 ${ }^{2}$ are very likely to have no translational symmetry, which would destroy the periodicity of the LOSC Hamiltonian, and the Bloch theorem is not applicable in principle. The examples of $\mathrm{C}_{4 n+2} \mathrm{H}_{4 n+2}$ show LOSC2 can gain more symmetry for LOs if the space allows. Wave funtions in bulk systems are normally delocalized over a large physical space, thus they tend to mix with each other into LOs that possess the translational symmetry of primitive cell or some supercell, which thus makes the Bloch theorem applicable.

\section{Some relations between symmetric localized orbitals}

For a set of LOs, $\left\{\phi_{p}\right\}$, they will remain orthonormal after applying any symmetry operator, i.e.

$$
\left\langle\phi_{p}\left|\hat{R}_{k}^{\dagger} \hat{R}_{k}\right| \phi_{q}\right\rangle=\left\langle\phi_{p} \mid \phi_{q}\right\rangle=\delta_{p q},
$$


thus $\hat{R}_{k}^{\dagger} \hat{R}_{k}=E$ and $\hat{R}_{k}^{\dagger}=\hat{R}_{k}^{-}$. If $\hat{R}_{k}$ is from the symmetry group of the system under study, and the two LOs, $\phi_{p^{\prime}}$ and $\phi_{p}$, obey the symmetry relation,

$$
\phi_{p^{\prime}}=\hat{R}_{k} \phi_{p}
$$

then for a one-electron Hamiltonian that satisfies Eq. (S1), the LO energies obey

$$
\left\langle\phi_{p^{\prime}}|\hat{h}| \phi_{p^{\prime}}\right\rangle=\left\langle\phi_{p}\left|\hat{R}_{k}^{\dagger} \hat{h} \hat{R}_{k}\right| \phi_{p}\right\rangle=\left\langle\phi_{p}\left|\hat{R}_{k}^{\dagger} \hat{R}_{k} \hat{h}\right| \phi_{p}\right\rangle=\left\langle\phi_{p}|\hat{h}| \phi_{p}\right\rangle
$$

Normally, the COs of the same irreducible representation are either all occupied or unoccupied, thus any occupied CO with $\hat{R}_{k}^{\dagger}$ applying on it can be obtained by linear combination of the occupied COs, i.e.

$$
\hat{R}_{k}^{\dagger}\left[\varphi_{1}(\mathbf{r}), \varphi_{2}(\mathbf{r}), \ldots, \varphi_{N}(\mathbf{r})\right]=\left[\varphi_{1}(\mathbf{r}), \varphi_{2}(\mathbf{r}), \ldots, \varphi_{N}(\mathbf{r})\right] \mathrm{U}^{\mathrm{k}}
$$

where $\mathrm{U}^{\mathrm{k}}$ is a unitary matrix. Therefore, the density matrix obeys

$$
\hat{R}_{k}^{\dagger} \hat{\rho} \hat{R}_{k}=\sum_{i=1}^{N} \hat{R}_{k}^{\dagger}\left|\varphi_{i}\right\rangle\left\langle\varphi_{i}\left|\hat{R}_{k}=\sum_{i, j, l=1}^{N}\right| \varphi_{j}\right\rangle U_{j i}^{k} U_{l i}^{k *}\left\langle\varphi_{l}\left|=\sum_{j, l=1}^{N}\right| \varphi_{j}\right\rangle \delta_{j l}\left\langle\varphi_{l}\left|=\sum_{j=1}^{N}\right| \varphi_{j}\right\rangle\left\langle\varphi_{j}\right|=\hat{\rho}
$$

and the occupations of two LOs satisfying Eq. (S55) are the same, i.e.

$$
\lambda_{p^{\prime} p^{\prime}}=\left\langle\phi_{p^{\prime}}|\hat{\rho}| \phi_{p^{\prime}}\right\rangle=\left\langle\phi_{p}\left|\hat{R}_{k}^{\dagger} \hat{\rho} \hat{R}_{k}\right| \phi_{p}\right\rangle=\left\langle\phi_{p}|\hat{\rho}| \phi_{p}\right\rangle=\lambda_{p p}
$$

According to Eq. (S56) and Eq. (S59), the symmetry of LOs can be analyzed by comparing the LO energies and occupations. In addition, let $\phi_{p}$ and $\phi_{p^{\prime}}$ be another pair of LOs that satisfy Eq. (S55), the off-diagonal element of local occupation matrix obeys

$$
\lambda_{p^{\prime} q^{\prime}}=\left\langle\phi_{p^{\prime}}|\hat{\rho}| \phi_{q^{\prime}}\right\rangle=\left\langle\phi_{p}\left|\hat{R}_{k}^{\dagger} \hat{\rho} \hat{R}_{k}\right| \phi_{q}\right\rangle=\left\langle\phi_{p}|\hat{\rho}| \phi_{q}\right\rangle=\lambda_{p q}
$$




\section{Supplemental test results}

In this work, DFT calculations were performed using an in-house developed $\mathrm{QM}^{4} \mathrm{D}$ program, ${ }^{3}$ while coupled cluster calculations using Gaussian09 program. ${ }^{4}$ IPs and EAs of G2-97 set, polyacenes and trans-polyacetylenes shown in Tabs S5-S7 were used to optimize the parameter $\gamma$ in Eq. 7. Two values of $\gamma(0.707$ and 0.780$)$ were obtained. The value 0.707 is used for all the tests in this work, while some test results for $\gamma=0.780$ are also provided here. The basis sets used are 6-311++G(3df, 3pd $)^{5-7}$ for HTBH38/08, NHTBH38/08, ${ }^{8,9}$ and G2-97 test sets; ${ }^{10}$ cc-pVTZ ${ }^{11}$ for polyacene oligomers, allenes, triphenylene, $\mathrm{H}_{2}^{+}, \mathrm{He}_{2}^{+}$, and $\mathrm{Li}_{2}^{+}$; cc-pVDZ for trans-polyacetylene oligomers. The minimal basis set is used for orbital density plots for $\mathrm{C}_{4 n+2} \mathrm{H}_{4 n+2}$ (both $\mathrm{C}_{6} \mathrm{H}_{6}$ and $\mathrm{C}_{10} \mathrm{H}_{10}$ ), while cc-pVTZ is used for IP calculations of $\mathrm{C}_{10} \mathrm{H}_{10}$. For LOSC1/LOSC2 calculations, only those COs with energies range from -30 to $10 \mathrm{eV}$ are taken into account for orbital localization and subsequent procedures, which makes the computational cost of the LOSC1/LOSC2 corrections much smaller than that of calculating SCF-converged COs.

Table S5: Mean absolute deviations (MDAs) computed for atomization energies (in $\mathrm{kcal} / \mathrm{mol}$ ), reaction barriers (in $\mathrm{kcal} / \mathrm{mol}$ ), and IPs and EAs (in eV).

\begin{tabular}{|c|c|c|c|c|c|c|c|c|c|c|c|c|}
\hline \multirow[b]{2}{*}{ Test sets ${ }^{\mathrm{a}}$} & \multicolumn{3}{|c|}{ DFA } & \multicolumn{3}{|c|}{ LOSC1-DFA } & \multicolumn{3}{|c|}{ LOSC2-DFA } & \multicolumn{3}{|c|}{ LOSC2-DFA $^{\mathrm{b}}$} \\
\hline & LDA & BLYP & B3LYP & LDA & BLYP & B3LYP & LDA & BLYP & B3LYP & LDA & BLYP & B3LYP \\
\hline \multicolumn{13}{|l|}{ Atomization energies $^{c}$} \\
\hline G2-1(55) & 35.15 & 4.97 & 2.70 & 35.59 & 4.95 & 2.79 & 35.14 & 4.97 & 2.70 & 35.14 & 4.97 & 2.70 \\
\hline Non-hydrogen(21) & 89.11 & 14.07 & 7.42 & 88.89 & 13.94 & 7.42 & 89.10 & 14.07 & 7.42 & 89.10 & 14.07 & 7.42 \\
\hline HydroCarbons(17) & 140.97 & 10.34 & 3.46 & 140.89 & 10.44 & 3.48 & 140.96 & 10.36 & 3.47 & 140.96 & 10.36 & 3.47 \\
\hline Substituted Hydrocarbons(42) & 118.67 & 6.23 & 2.52 & 118.58 & 6.27 & 2.54 & 118.65 & 6.24 & 2.53 & 118.65 & 6.24 & 2.53 \\
\hline Inorganic hydrides and radicals(13) & 73.01 & 5.41 & 2.26 & 73.42 & 5.54 & 2.15 & 72.99 & 5.41 & 2.26 & 72.99 & 5.41 & 2.26 \\
\hline mean $(148)$ & 81.99 & 7.28 & 3.37 & 82.12 & 7.28 & 3.40 & 81.97 & 7.28 & 3.37 & 81.97 & 7.28 & 3.37 \\
\hline \multicolumn{13}{|l|}{ Reaction barriers $^{\mathrm{d}}$} \\
\hline $\operatorname{HTBH}(38)$ & 17.35 & 7.60 & 4.34 & 16.30 & 6.82 & 4.31 & 17.03 & 7.42 & 4.34 & 17.22 & 7.54 & 4.34 \\
\hline NHTBH(38) & 12.49 & 8.57 & 4.56 & 11.75 & 7.90 & 4.53 & 12.50 & 8.60 & 4.56 & 12.50 & 8.60 & 4.56 \\
\hline mean $(76)$ & 14.92 & 8.09 & 4.45 & 14.03 & 7.36 & 4.42 & 14.76 & 8.01 & 4.45 & 14.86 & 8.07 & 4.45 \\
\hline \multicolumn{13}{|l|}{ IPs \& EAs ${ }^{\mathrm{e}}$} \\
\hline $\operatorname{IP}(64)$ & 4.46 & 4.50 & 3.19 & 0.50 & 0.63 & 0.37 & 0.50 & 0.63 & 0.35 & 0.50 & 0.63 & 0.35 \\
\hline $\mathrm{EA}(47)$ & 3.79 & 3.40 & 2.61 & 0.50 & 0.52 & 0.48 & 0.49 & 0.50 & 0.48 & 0.49 & 0.50 & 0.48 \\
\hline mean $(111)$ & 4.18 & 4.03 & 2.94 & 0.50 & 0.58 & 0.42 & 0.50 & 0.57 & 0.40 & 0.50 & 0.57 & 0.40 \\
\hline \multicolumn{13}{|c|}{ a The number in parentheses indicates the number of systems tested. } \\
\hline \multirow{2}{*}{\multicolumn{13}{|c|}{$\begin{array}{l}\text { b LOSC2 calculations with the parameter } \gamma \text { in the objective function Eq. (7) set to } 0.780 \text {. } \\
\text { c Atomization energies are tested on the G2-97 test set. Geometries and experimental date }\end{array}$}} \\
\hline & & & & & & & & & & & & \\
\hline \multicolumn{13}{|c|}{$\begin{array}{l}\text { d Reaction barriers are tested on the HTBH38/08 and NHTBH38/08 test sets. Geometries and experimental data are from refs. } 8,9 \text {. } \\
\text { e The testing molecules are from the G2-97 set, } \mathrm{H}_{2} \mathrm{~S}\left({ }^{2} \mathrm{~A}_{1}\right), \mathrm{N}_{2}\left({ }^{2} \Pi_{u}\right), \mathrm{C}_{6} \mathrm{H}_{6}, \mathrm{C}_{7} \mathrm{H}_{8}, \mathrm{H}_{6} \mathrm{C}_{6} \mathrm{O} \text { and } \mathrm{H}_{7} \mathrm{C}_{6} \mathrm{O} \text { are exclusive from the IP calculations. Reference }\end{array}$} \\
\hline $\begin{array}{l}\text { e The testing molecules are fron } \\
\text { data are obtained by } \operatorname{CCSD}(\mathrm{T}\end{array}$ & 007 & & 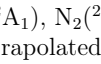 & & & & 0 & & e fron & & & \\
\hline
\end{tabular}


Table S6: HOMO and LUMO energies of polyacene oligomers calculated by different DFT methods. Experimental data are included for comparison. All energies are in eV.

\begin{tabular}{|c|c|c|c|c|c|c|c|c|c|c|c|c|c|c|}
\hline & \multirow[b]{2}{*}{$\mathrm{N}$} & \multicolumn{3}{|c|}{ DFA } & \multicolumn{3}{|c|}{ LOSC1-DFA $^{a}$} & \multicolumn{3}{|c|}{ LOSC2-DFA } & \multicolumn{3}{|c|}{ LOSC2-DFA $^{b}$} & \multirow[b]{2}{*}{ Expt. ${ }^{c}$} \\
\hline & & LDA & BLYP & B3LYP & LDA & BLYP & B3LYP & LDA & $\overline{B L Y P}$ & B3LYP & LDA & BLYP & B3LYP & \\
\hline \multirow{7}{*}{ HOMO } & 1 & -6.50 & -6.09 & -7.04 & -8.93 & -8.51 & -8.96 & -8.90 & -8.49 & -8.96 & -8.90 & -8.50 & -8.95 & -9.24 \\
\hline & 2 & -5.68 & -5.26 & -6.11 & -8.16 & -7.71 & -8.05 & -8.60 & -8.18 & -8.40 & -8.21 & -7.80 & -8.06 & -8.11 \\
\hline & 3 & -5.19 & -4.76 & -5.54 & -7.66 & -7.23 & -7.36 & -7.84 & -7.42 & -7.56 & -7.64 & -7.22 & -7.30 & -7.47 \\
\hline & 4 & -4.87 & -4.44 & -5.16 & -7.21 & -6.80 & -6.89 & -7.45 & -7.03 & -7.18 & -7.16 & -6.75 & -6.80 & -6.97 \\
\hline & 5 & -4.66 & -4.22 & -4.90 & -6.82 & -6.42 & -6.46 & -6.91 & -6.73 & -6.62 & -6.89 & -6.41 & -6.53 & -6.63 \\
\hline & 6 & -4.50 & -4.06 & -4.71 & -6.62 & -6.15 & -6.24 & -6.98 & -6.54 & -6.26 & -6.64 & -6.20 & -6.08 & -6.36 \\
\hline & MAE $^{\mathrm{d}}$ & 2.23 & 2.66 & 1.89 & 0.21 & 0.33 & 0.14 & 0.43 & 0.20 & 0.17 & 0.22 & 0.32 & 0.18 & \\
\hline \multirow{7}{*}{ LUMO } & 1 & -1.38 & -0.97 & -0.39 & 0.71 & 1.12 & 1.25 & 0.73 & 1.11 & 1.26 & 0.73 & 1.10 & 1.26 & 1.12 \\
\hline & 2 & -2.25 & -1.82 & -1.33 & -0.16 & 0.26 & 0.31 & -0.15 & 0.28 & 0.35 & -0.16 & 0.27 & 0.31 & -0.20 \\
\hline & 3 & -2.83 & -2.39 & -1.97 & -0.77 & -0.33 & -0.37 & -0.68 & -0.24 & -0.33 & -0.75 & -0.30 & -0.34 & -0.53 \\
\hline & 4 & -3.22 & -2.76 & -2.41 & -1.26 & -0.78 & -0.94 & -1.11 & -0.65 & -0.77 & -1.18 & -0.72 & -0.91 & -1.06 \\
\hline & 5 & -3.48 & -3.02 & -2.71 & -1.59 & -1.13 & -1.38 & -1.38 & -0.92 & -1.09 & -1.49 & -1.03 & -1.23 & -1.39 \\
\hline & 6 & -3.67 & -3.21 & -2.93 & -1.77 & -1.32 & -1.52 & -1.60 & -1.13 & -1.32 & -1.75 & -1.27 & -1.49 & -1.47 \\
\hline & $\mathrm{MAE}^{\mathrm{d}}$ & 2.22 & 1.78 & 1.37 & 0.23 & 0.22 & 0.16 & 0.13 & 0.33 & 0.27 & 0.19 & 0.27 & 0.20 & \\
\hline
\end{tabular}

a The LOSC1-DFA results are from ref. 2.

b LOSC2 calculations with the parameter $\gamma$ in the objective function Eq. (7) set to 0.780 .

c The experimental values are from refs. 12-14.

d The mean absolute error for each method as compared to the experimental values.

Table S7: HOMO energies, $\varepsilon_{\mathrm{HOMO}}$, of trans-polyacetylene oligomers, $\mathrm{H}(\mathrm{HC}=\mathrm{CH})_{n} \mathrm{H}$, calculated by different DFT methods. RASPT2 and experimental data are included for comparison. All energies are in $\mathrm{eV}$.

\begin{tabular}{|c|c|c|c|c|c|c|c|c|c|c|c|c|c|c|}
\hline \multirow[b]{2}{*}{$n$} & \multicolumn{3}{|c|}{ DFA } & \multicolumn{3}{|c|}{ LOSC1-DFA } & \multicolumn{3}{|c|}{ LOSC2-DFA } & \multicolumn{3}{|c|}{ LOSC2-DFA $^{a}$} & \multirow{2}{*}{ RASPT $^{\mathrm{b}}$} & \multirow{2}{*}{ Expt. $^{\mathrm{c}}$} \\
\hline & LDA & BLYP & B3LYP & LDA & BLYP & B3LYP & LDA & BLYP & B3LYP & LDA & BLYP & B3LYP & & \\
\hline 1 & -6.71 & -6.34 & -7.46 & -10.46 & -10.10 & -10.46 & -10.46 & -10.10 & -10.46 & -10.46 & -10.11 & -10.46 & -10.48 & -10.51 \\
\hline 2 & -5.84 & -5.46 & -6.43 & -9.01 & -8.66 & -8.75 & -9.48 & -9.12 & -9.23 & -8.23 & -7.86 & -8.35 & -9.18 & -9.07 \\
\hline 3 & -5.40 & -5.01 & -5.89 & -8.26 & -7.78 & -7.98 & -8.44 & -8.47 & -8.04 & -8.35 & -8.07 & -7.79 & -8.18 & -8.29 \\
\hline 4 & -5.14 & -4.74 & -5.56 & -7.98 & -7.24 & -7.61 & -8.05 & -7.70 & -7.80 & -7.71 & -7.34 & -7.56 & -7.69 & -7.79 \\
\hline 5 & -4.97 & -4.56 & -5.34 & -7.33 & -6.94 & -7.23 & -7.82 & -7.67 & -7.55 & -7.62 & -7.23 & -7.14 & -7.33 & \\
\hline 6 & -4.84 & -4.43 & -5.17 & -7.31 & -6.92 & -7.05 & -7.63 & -7.30 & -7.32 & -7.30 & -6.89 & -7.10 & -7.04 & \\
\hline 7 & -4.75 & -4.33 & -5.05 & -7.21 & -6.63 & -6.84 & -7.59 & -7.33 & -7.29 & -7.19 & -6.79 & -6.88 & -6.85 & \\
\hline 8 & -4.68 & -4.25 & -4.95 & -6.95 & -6.55 & -6.74 & -7.34 & -6.97 & -7.03 & -7.09 & -6.64 & -6.83 & -6.66 & \\
\hline 9 & -4.62 & -4.19 & -4.87 & -6.81 & -6.40 & -6.61 & -7.14 & -6.76 & -6.93 & -6.92 & -6.52 & -6.62 & -6.56 & \\
\hline 10 & -4.57 & -4.14 & -4.80 & -6.75 & -6.35 & -6.60 & -7.13 & -6.64 & -6.90 & -6.80 & -6.40 & -6.53 & -6.41 & \\
\hline$\infty^{\mathrm{d}}$ & -3.52 & -3.06 & -3.47 & -4.91 & -4.45 & -4.76 & -5.27 & -4.77 & -5.04 & -5.27 & -4.83 & -5.08 & -4.56 & \\
\hline $\mathrm{MAE}_{1}{ }^{\mathrm{e}}$ & 2.36 & 2.77 & 2.00 & 0.22 & 0.26 & 0.13 & 0.50 & 0.25 & 0.27 & 0.36 & 0.26 & 0.23 & & \\
\hline $\mathrm{MAE}_{2}{ }^{\mathrm{f}}$ & 3.14 & 3.53 & 2.58 & 0.08 & 0.47 & 0.22 & 0.22 & 0.18 & 0.12 & 0.26 & 0.57 & 0.37 & 0.09 & \\
\hline
\end{tabular}

${ }^{a}$ LOSC2 calculations with the parameter $\gamma$ in the objective function Eq. (7) set to 0.780.

$\mathrm{b}$ The second order restrictive active space perturbation theory values are from ref. 15

c The experimental values for $n=1-4$ are from refs. $16-18$

${ }^{d}$ IPs at $n \rightarrow \infty$ are obtained by extrapolation as shown in Fig. S2.

e The mean absolute error for each method as compared to the RASPT2 values.

f The mean absolute error for each method as compared to the experimental values. 


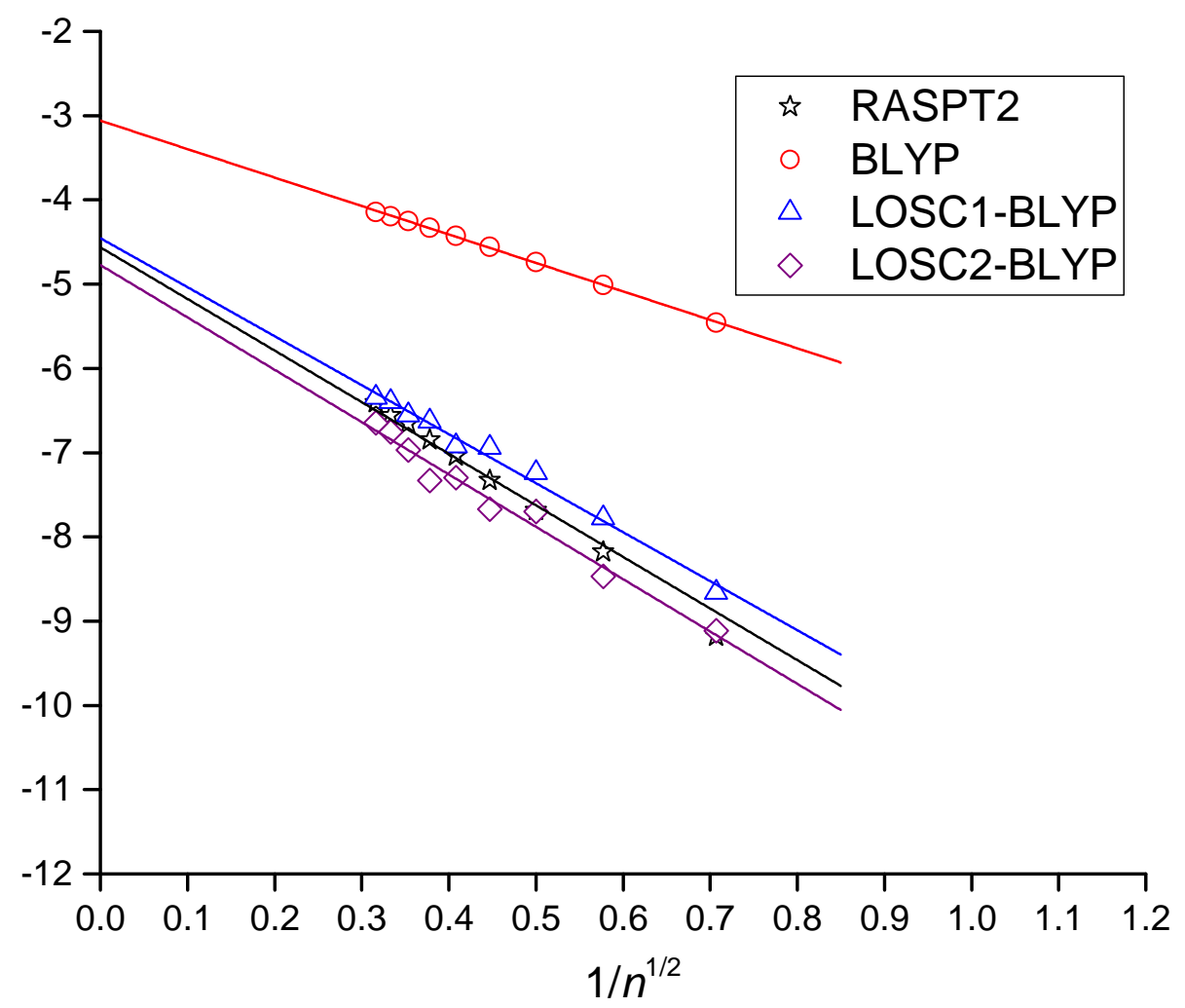

Figure S2: Extrapolation from calculated HOMO energies for trans-polyacetylene oligomers $\mathrm{H}(\mathrm{HC}=\mathrm{CH})_{n} \mathrm{H}$ with $n \leq 10$ to $n \rightarrow \infty$. As the calculated data for HOMO energies exhibit linear behavior against $1 / n^{1 / 2}$. By fitting the data to straight lines and then extrapolating to $1 / n^{1 / 2}=0$, the bulk values of IPs may be estimated. 


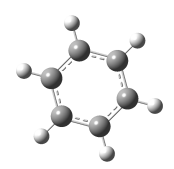

(a) BLYP
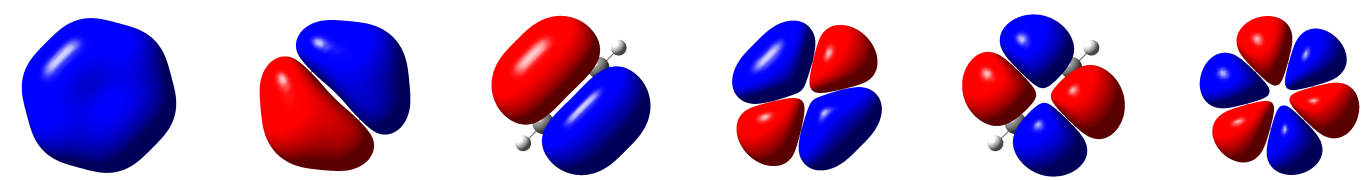

$\mathrm{e}_{2 \mathrm{u}}(0.00)$

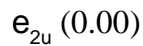

$b_{2 g}(0.00)$

(b) LOSC1-BLYP

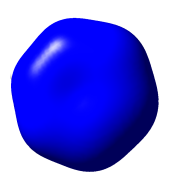

I (1.00)
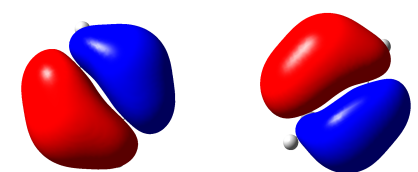

III (1.00)

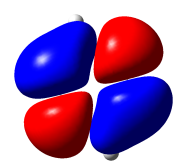

IV (0.00)
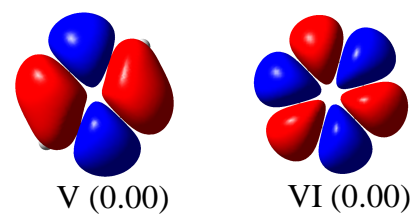

(c) LOSC2-BLYP

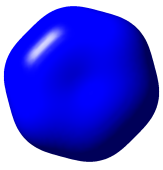

I (1.00)
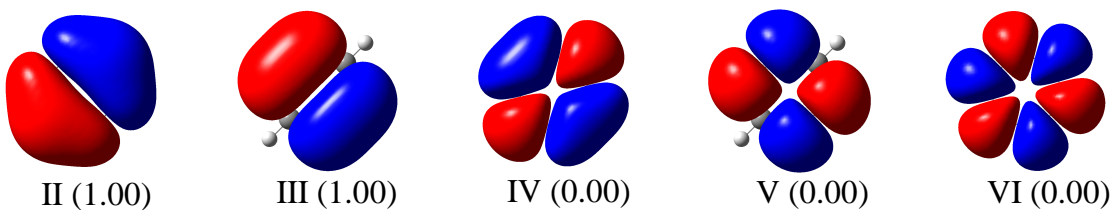

Figure S3: $\pi$-bond COs and LOs from the mix of them for benzene $\left(\mathrm{C}_{6} \mathrm{H}_{6}\right.$ with $R_{\mathrm{CC}}=1.50$ and $\left.R_{\mathrm{HH}}=1.09, D_{6 h}\right)$. The values in the parentheses are the occupations of COs and LOs, $\langle\hat{\rho}\rangle$. Minimal basis set is used. 


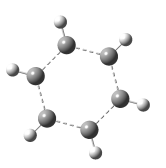

(a) BLYP
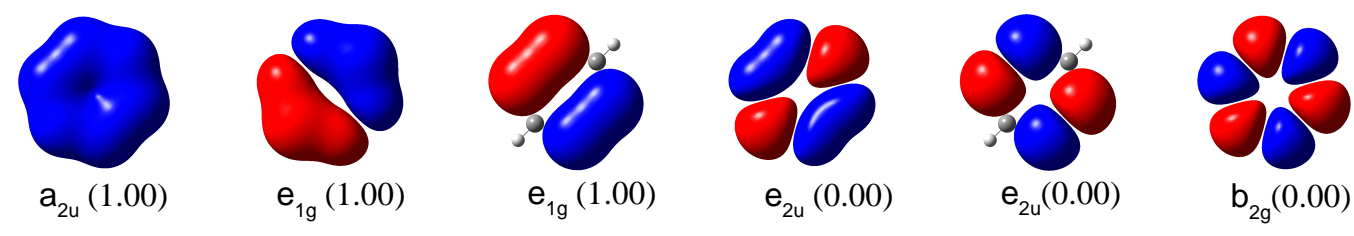

$\mathrm{a}_{2 \mathrm{u}}(1.00)$

$\mathrm{e}_{1 \mathrm{~g}}(1.00)$

$\mathrm{e}_{1 \mathrm{~g}}(1.00)$
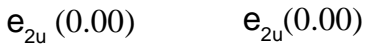

$\mathrm{b}_{2 \mathrm{~g}}(0.00)$

(b) LOSC1-BLYP
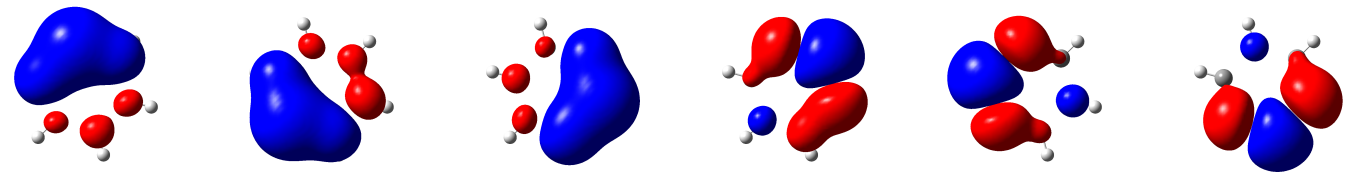

(c) LOSC2-BLYP

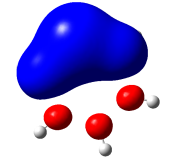

I (0.83)

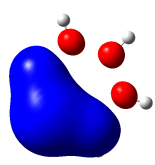

II $(0.83)$

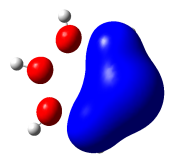

III (0.83)

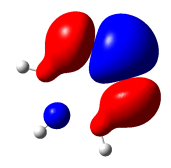

IV (0.17)

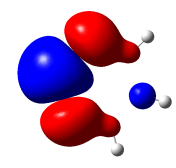

$\mathrm{V}(0.17)$

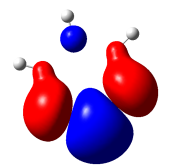

VI (0.17)

Figure S4: $\pi$-bond COs and LOs from the mix of them for stretched benzene $\left(\mathrm{C}_{6} \mathrm{H}_{6}\right.$ with $R_{\mathrm{CC}}=2.00$ and $\left.R_{\mathrm{HH}}=1.09, D_{6 h}\right)$. The values in the parentheses are the occupations of COs and LOs, $\langle\hat{\rho}\rangle$. Minimal basis set is used. 


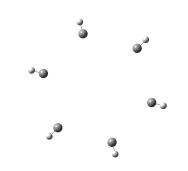

(a) BLYP

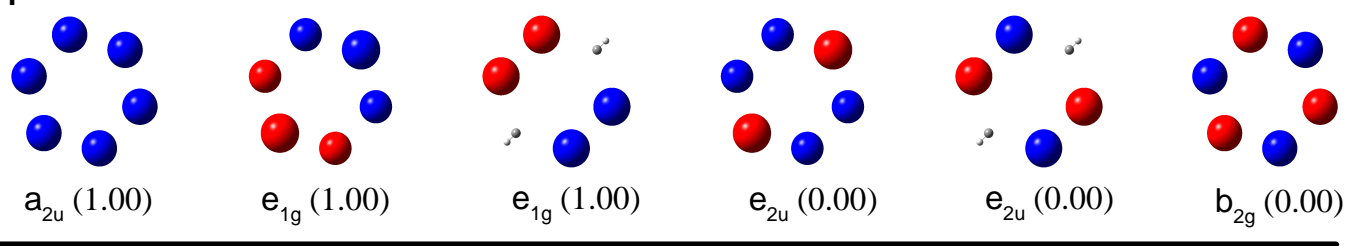

(b) LOSC1-BLYP

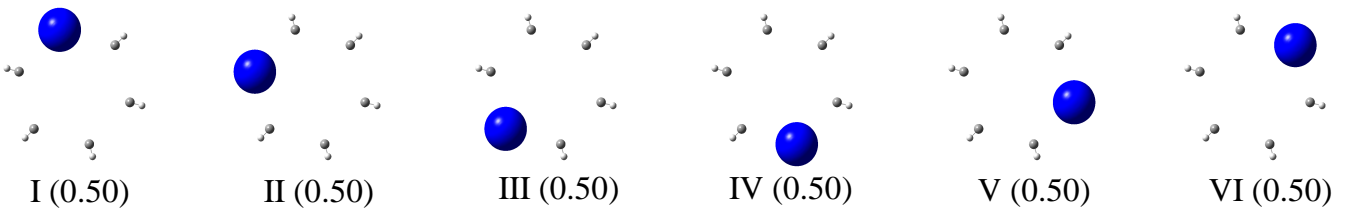

(c) LOSC2-BLYP
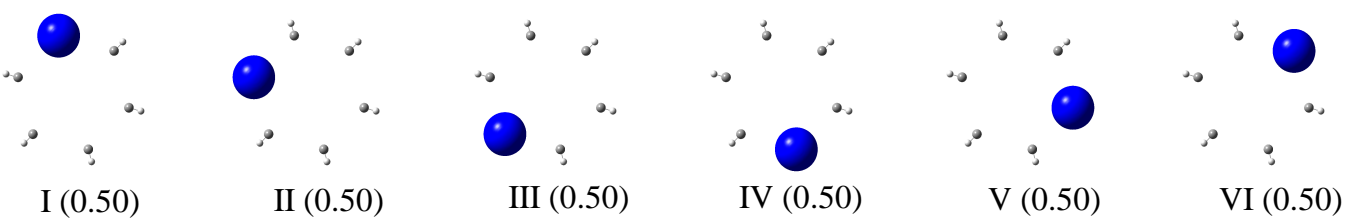

Figure S5: $\pi$-bond COs and LOs from the mix of them for overstretched benzene $\left(\mathrm{C}_{6} \mathrm{H}_{6}\right.$ with $R_{\mathrm{CC}}=5.00$ and $\left.R_{\mathrm{HH}}=1.09, D_{6 h}\right)$. The values in the parentheses are the occupations of COs and LOs, $\langle\hat{\rho}\rangle$. Minimal basis set is used. 


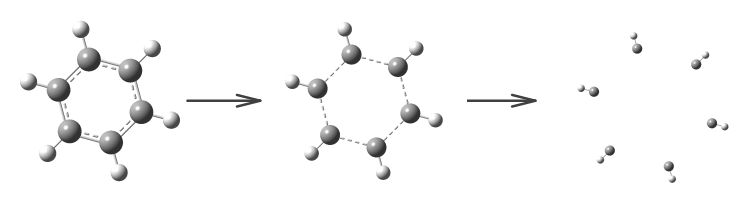

(a) $R_{\mathrm{CC}}=1.5 \AA$
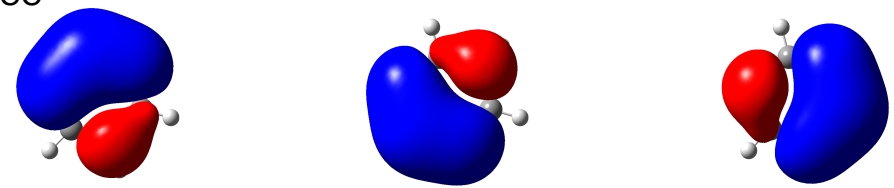

I (1.00)

II (1.00)

III (1.00)

(b) $R_{\mathrm{CC}}=2.0 \AA$
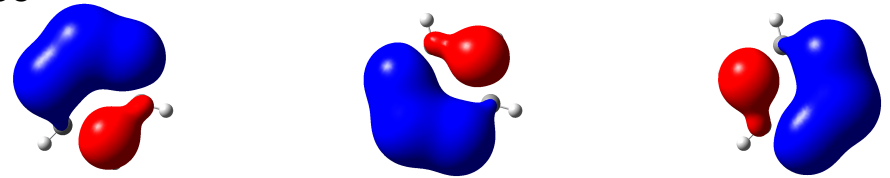

I (1.00)

II (1.00)

III (1.00)

(c) $R_{\mathrm{CC}}=5.0 \AA$

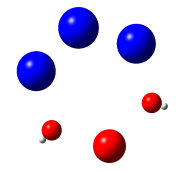

I (1.00)
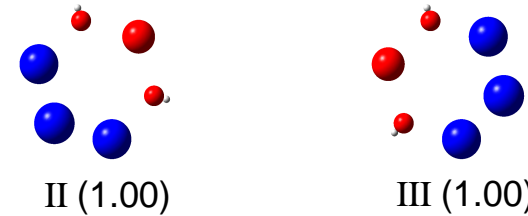

III (1.00)

Figure S6: LOs from the mix of $\pi$-bonding COs by the Foster-Boys localization. Benzenes $\left(\mathrm{C}_{6} \mathrm{H}_{6}, D_{6 h}\right)$ with $R_{\mathrm{C}-\mathrm{C}}=1.5,2.0$, and 5.0 are tested. The values in the parentheses are the occupations of COs and LOs, $\langle\hat{\rho}\rangle$. As only occupied COs are mixed, all the occupations are 1.0. Minimal basis set is used. 


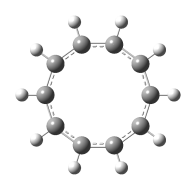

(a) BLYP
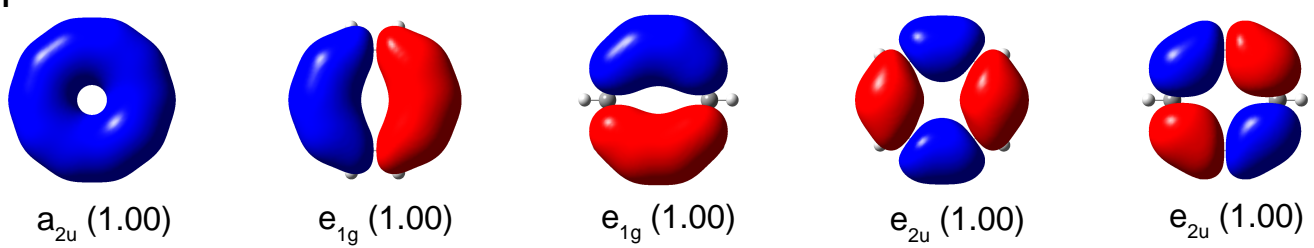

$\mathrm{e}_{2 \mathrm{u}}(1.00)$

$\mathrm{e}_{2 \mathrm{u}}(1.00)$
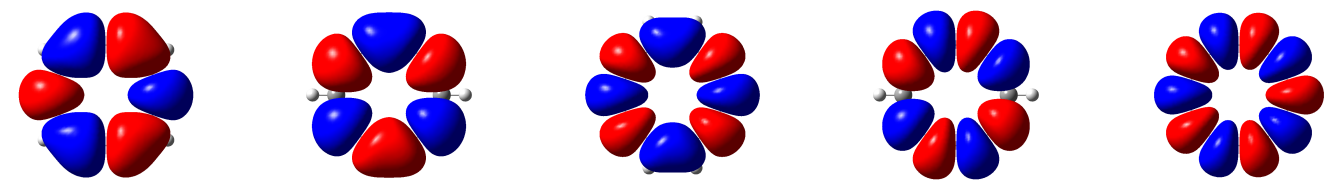

$e_{2 g}(0.00)$

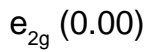

$\mathrm{e}_{1 \mathrm{u}}(0.00)$

$\mathrm{e}_{1 \mathrm{u}}(0.00)$

$a_{1 g}(0.00)$

(b) LOSC1-BLYP

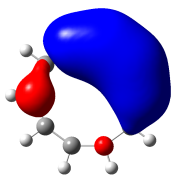

I (1.00)

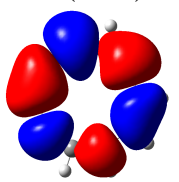

VI (0.07)

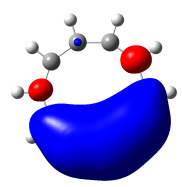

II (0.99)

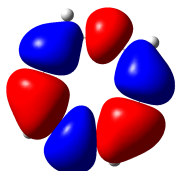

VII (0.04)

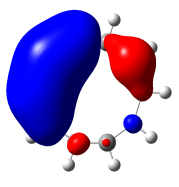

III (1.00)

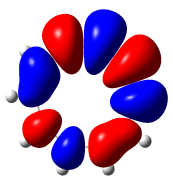

VIII (0.00)

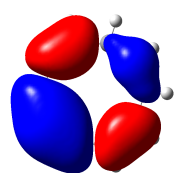

IV (0.96)

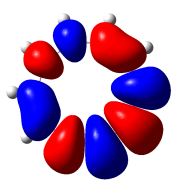

IX $(0.00)$

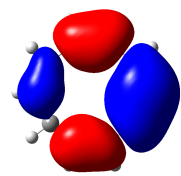

V (0.94)

(c) LOSC2-BLYP

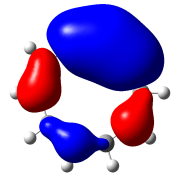

I (0.98)

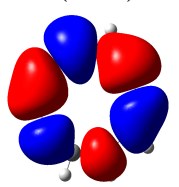

VI (0.05)

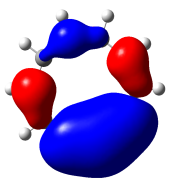

II (0.98)

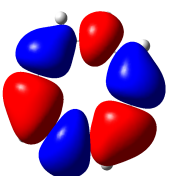

VII (0.05)

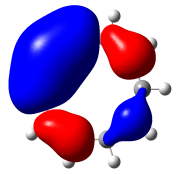

III (0.98)

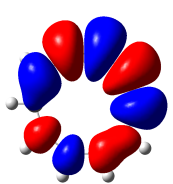

VIII (0.00)

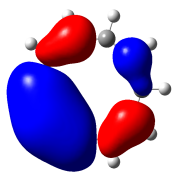

IV (0.98)

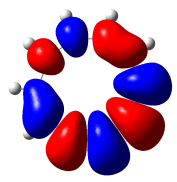

IX (0.00)

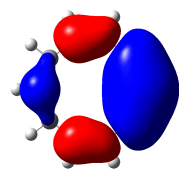

V (0.98)

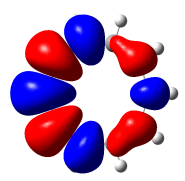

X (0.00)

Figure S7: $\pi$-bond COs and LOs from the mix of them for planar [10]annulene $\left(\mathrm{C}_{10} \mathrm{H}_{10}\right.$ with $R_{\mathrm{CC}}=1.50$ and $\left.R_{\mathrm{HH}}=1.09, D_{10 h}\right)$. The values in the parentheses are the occupations of COs and LOs, $\langle\hat{\rho}\rangle$. Minimal basis set is used. 

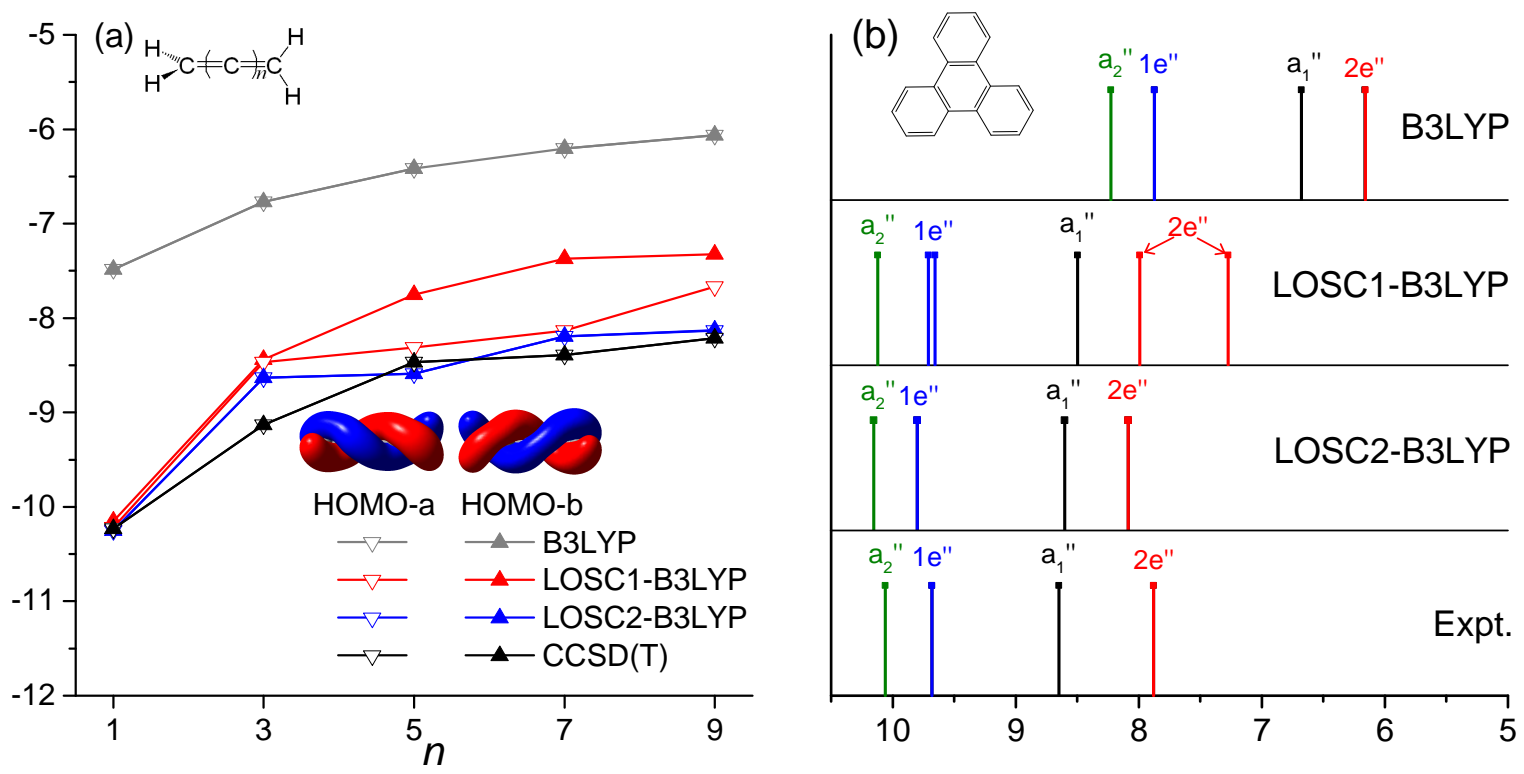

Figure S8: Comparison of the energy level and state degeneracy among B3LYP, LOSC1B3LYP, and LOSC2-B3LYP. (a) HOMOs of odd numbered allenes are tested. Odd numbered allenes have doubly degenerate HOMOs, which are helical and of symmetry e, hence they are achiral. Misprediction of the degeneracy of the two HOMOs would lead to misjudgment of the molecular chirality. The y axis represents orbital energy in eV. The CCSD(T) results are used as reference. (b) Vertical ionization potentials of triphenylene $\left(D_{3 h}\right)$ are tested, where $1 \mathrm{e}^{\prime \prime}$ and $2 \mathrm{e}^{\prime \prime}$ are two pairs of doubly degenerate states. The $\mathrm{x}$ axis represents IPs in $\mathrm{eV}$. Experimental results are from ref. 19. All geometries are optimized by B3LYP. 

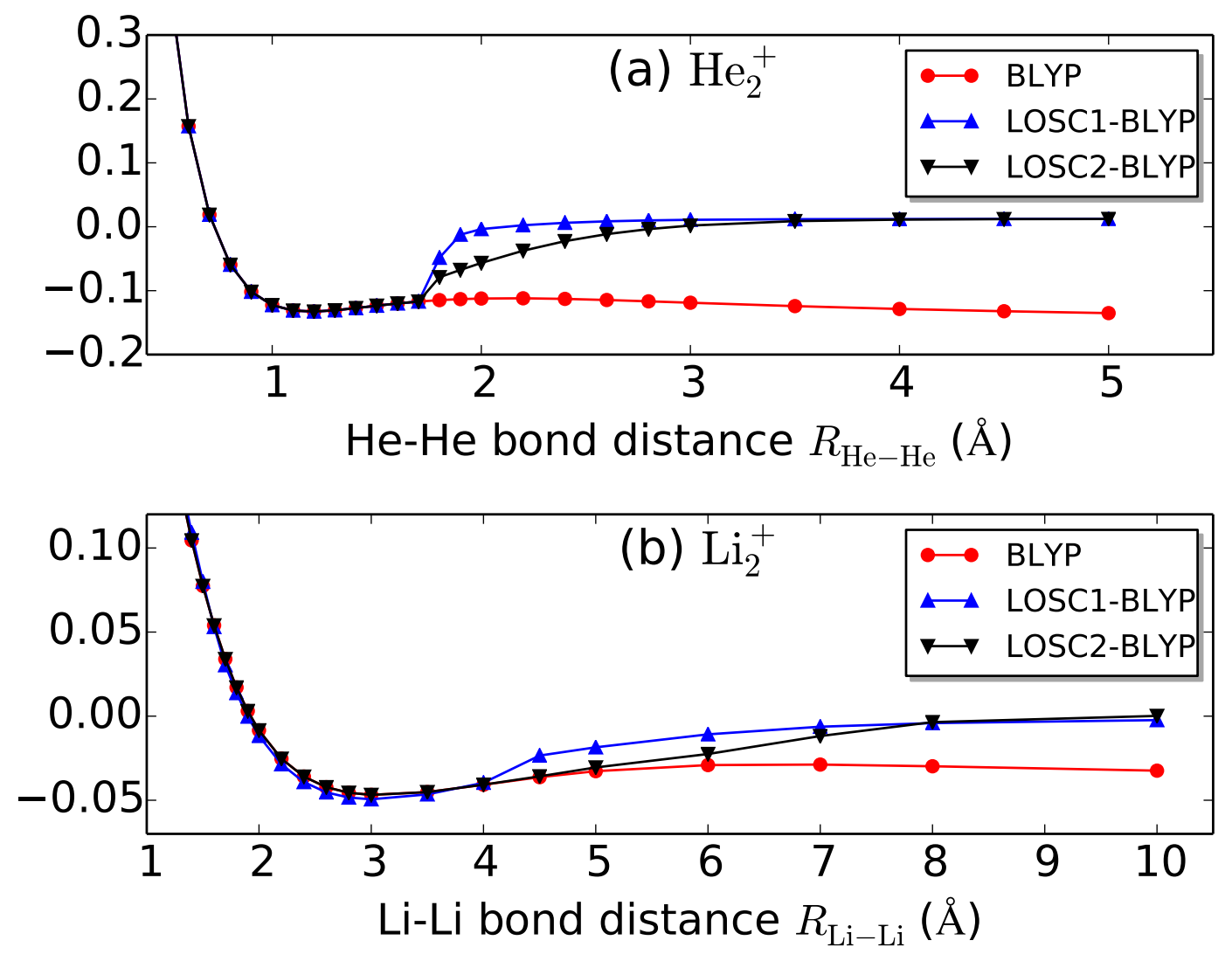

Figure S9: (a). Potential energy curves for $\mathrm{He}_{2}^{+}$dissociation. The total energy of a singlet $\mathrm{He}$ atom and a doublet $\mathrm{He}^{+}$cation is set to zero. (b). Potential energy curves for $\mathrm{Li}_{2}^{+}$ dissociation. The total energy of a doublet $\mathrm{Li}$ atom and a singlet $\mathrm{Li}^{+}$cation is set to zero. All energies are in atomic units. LOSC2 improves the performance of LOSC1, but the curves still show derivative discontinuity at the points where HOMO and LUMO begin to mix together into two symmetric LOs. 


\section{References}

(1) Ashcroft, N.; Mermin, N. Solid State Physics; HRW international editions; Holt, Rinehart and Winston, 1976.

(2) Li, C.; Zheng, X.; Su, N. Q.; Yang, W. Localized Orbital Scaling Correction for Systematic Elimination of Delocalization Error in Density Functional Approximations. Nat. Sci. Rev. 2017, nwx111.

(3) QM4D, An in-house program for QM/MM simulations (http://www.qm4d.info). 2019.

(4) Frisch, M. J. et al. Gaussian 09 Revision A.1. 2009.

(5) Krishnan, R.; Binkley, J. S.; Seeger, R.; Pople, J. A. Self-Consistent Molecular Orbital Methods. XX. A Basis Set for Correlated Wave Functions. J. Chem. Phys. 1980, 72, $650-654$.

(6) Frisch, M. J.; Pople, J. A.; Binkley, J. S. Self-Consistent Molecular Orbital Methods 25. Supplementary Functions for Gaussian Basis Sets. J. Chem. Phys. 1984, 80, 3265-3269.

(7) Clark, T.; Chandrasekhar, J.; Spitznagel, G. W.; Schleyer, P. V. R. Efficient Diffuse Function-Augmented Basis Sets for Anion Calculations. III. The 3-21+G Basis Set for First-Row Elements, Li-F. J. Comput. Chem. 1983, 4, 294-301.

(8) Zheng, J.; Zhao, Y.; Truhlar, D. G. The DBH24/08 Database and Its Use to Assess Electronic Structure Model Chemistries for Chemical Reaction Barrier Heights. J. Chem. Theory Comput. 2009, 5, 808-821.

(9) Peverati, R.; Truhlar, D. G. Quest for a Universal Density Functional: the Accuracy of Density Functionals Across a Broad Spectrum of Databases in Chemistry and Physics. Philos. Trans. Royal Soc. A 2014, 372. 
(10) Curtiss, L. A.; Raghavachari, K.; Redfern, P. C.; Pople, J. A. Assessment of Gaussian-2 and Density Functional Theories for the Computation of Enthalpies of Formation. J. Chem. Phys. 1997, 106, 1063-1079.

(11) Jr., T. H. D. Gaussian Basis Sets for Use in Correlated Molecular Calculations. I. The Atoms Boron Through Neon and Hydrogen. J. Chem. Phys. 1989, 90, 1007-1023.

(12) Linstrom, P.; Mallard, W. Eds., NIST Chemistry WebBook, NIST Standard Reference Database Number 69, National Institute of Standards and Technology, Gaithersburg MD, 20899. See http://webbook.nist.gov 2017,

(13) Burrow, P. D.; Michejda, J. A.; Jordan, K. D. Electron Transmission Study of the Temporary Negative Ion States of Selected Benzenoid and Conjugated Aromatic Hydrocarbons. J. Chem. Phys. 1987, 86, 9-24.

(14) Hajgató, B.; Deleuze, M. S.; Tozer, D. J.; Proft, F. D. A Benchmark Theoretical Study of the Electron Affinities of Benzene and Linear Acenes. J. Chem. Phys. 2008, 129, 084308, see this reference for the best estimate of EA of hexacene.

(15) Shahi, A. R. M.; Cramer, C. J.; Gagliardi, L. Second-Order Perturbation Theory with Complete and Restricted Active Space Reference Functions Applied to Oligomeric Unsaturated Hydrocarbons. Phys. Chem. Chem. Phys. 2009, 11, 10964-10972, see this reference for experimental IPs of gas phase trans-polyacetylene oligomers for $N=1-4$ and RASPT2 results for $N=1-10$.

(16) Beez, M.; Bieri, G.; Bock, H.; Heilbronner, E. The Ionization Potentials of Butadiene, Hexatriene, and Their Methyl Derivatives: Evidence for Through Space Interaction Between Double Bond $\pi$-Orbitals and Non-Bonded Pseudo- $\pi$ Orbitals of Methyl Groups? Helvetica Chimica Acta 1973, 56, 1028-1046.

(17) Kimura, K. Handbook of HeI Photoelectron Spectra of Fundamental Organic Molecules: 
Ionization Energies, Ab Initio Assignments, and Valence Electronic Structure for 200 Molecules; Japan Scientific Societies Press, 1981.

(18) Bally, T.; Nitsche, S.; Roth, K.; Haselbach, E. Excited States of Polyene Radical Cations: Limitations of Koopmans' Theorem. J. Am. Chem. Soc. 1984, 106, 39273933.

(19) Schmidt, W. Photoelectron Spectra of Polynuclear Aromatics. V. Correlations with Ultraviolet Absorption Spectra in the Catacondensed Series. J. Chem. Phys. 1977, 66, $828-845$. 